5. Hein IM, Troost PW, De Vries MC, Knibbe CAJ, Van Goudoever JB, Lindauer RJL. Why do children decide not to participate in clinical research: A quantitative and qualitative study. Pediatr Res 2015;

6. Van Berge Henegouwen MTH, Van Driel HF, Kasteleijn-Nolst Trenité DGA. A patient diary as a tool to improve medicine compliance. Pharm World Sci 1999:21(1):21-4.

7. Stone AA. Patient non-compliance with paper diaries. BMJ 2002;

Disclosure(s) Nothing to disclose

\section{P60 SERUM CREATININE IS SUPERIOR TO SERUM CYSTATIN C IN EXPLAINING INTER-INDIVIDUAL VARIABILITY OF VANCOMYCIN CLEARANCE IN NEONATES AND YOUNG INFANTS}

${ }^{1,2} \mathrm{~S}$ Leroux ${ }^{*},{ }^{3} \mathrm{~V}$ Biran, ${ }^{1,4} \mathrm{~J}$ van den Anker, ${ }^{5} \mathrm{~W}$ Zhao, ${ }^{2} \mathrm{E}$ Jacqz-Aigrain* ${ }^{*}{ }^{1} \mathrm{M}$ Pfister ${ }^{*}$. 'Pediatric Pharmacology and Pharmacometrics, University Children's Hospital Basel, University of Basel, Basel, Switzerland; 'Department of Pediatric Pharmacology and Pharmacogenetics, Hôpital Robert Debré, APHP; ${ }^{3}$ Neonatal Intensive Care Unit, Hôpital Robert Debré, APHP, Paris, France; ${ }^{4}$ Division of Clinical Pharmacology, Children's National Health System, Washington, DC, USA; ${ }^{5}$ Department of Clinical Pharmacy, School of Pharmaceutical Sciences, Shandong University, Jinan, China

\subsection{6/archdischild-2019-esdppp.98}

Background Serum creatinine (SCr) is used as a surrogate marker of glomerular filtration rate to guide dosing of drugs eliminated by the kidney. Serum Cystatin C (S-CysC) has been suggested as a more reliable biomarker than SCr for monitoring kidney function in adults and children. Purpose of this study was to evaluate whether S-CysC is a more useful marker for estimating vancomycin clearance in neonates and young infants.

Methods Pharmacokinetic (PK), Scr and S-CysC data were collected in patients undergoing continuous intravenous vancomycin treatment in the neonatal intensive care unit of Robert Debré Hospital - Paris. A population PK analysis was performed with the software package NONMEM, utilizing vancomycin therapeutic drug monitoring samples. S-CysC and $\mathrm{SCr}$ were compared in terms of usefulness to explain inter-individual variability (IIV) of vancomycin clearance. Statistical criteria were used for covariate selection.

Results A total of 58 concentrations from 47 patients (gestational age [GA] ranging from 23 to 41 weeks and postmenstrual age [PMA] ranging from 26 to 46 weeks) were modeled with an allometric one-compartment model with first-order elimination. The median (range) values for $\mathrm{SCr}$ and S-CysC were 41.5 (12.0 to 102.0$) \mu \mathrm{mol} / \mathrm{L}$ and 1.38 (0.95 to 2.32 ) $\mathrm{mg} / \mathrm{L}$, respectively. When tested individually, SCr and S-CysC explained $26.4 \%$ and $9.8 \%$ of IIV of vancomycin clearance, respectively. PMA was superior as a covariate on clearance, over the combination of GA and postnatal age. As such, bodyweight, PMA, and SCr were retained as significant covariates on clearance in the final model.

Conclusions Serum Creatinine was found to be more useful than Serum Cystatin C to explain IIV of vancomycin clearance in this population. Further studies are needed to evaluate the utility of renal biomarkers to predict clearance and dosing of renally eliminated drugs in neonates.

Disclosure(s) Nothing to disclose

\section{P61 POPULATION PHARMACOKINETICS OF VANCOMYCIN IN CHINESE ICU NEONATES: INITIAL DOSAGE RECOMMENDATIONS}

'Z Li*, ${ }^{2} Z$ Jiao. 'Shanghai Children's Hospital, Pharmacy, Shanghai Children's Hospital;

${ }^{2}$ Huashan Hospital of Fudan University, Shanghai, China

10.1136/archdischild-2019-esdppp.99

The main goal of our study was to characterize the population pharmacokinetics of vancomycin in critically ill Chinese neonates to develop a pharmacokinetic model and investigate factors that have significant influences on the pharmacokinetics of vancomycin in this population. ${ }^{2}$ The study population consisted of 80neonates in the neonatal intensive care unit (ICU)from which 165 trough and peak concentrations of vancomycin were obtained.Nonlinear mixed effect modeling was used to develop a population pharmacokinetic model for vancomycin. ${ }^{4}$ The stability and predictive ability of the final model were evaluated based on diagnostic plots, normalized prediction distribution errorsandthe bootstrap method.Serum creatinine ( $\mathrm{Scr}$ ) and body weight were significant covariates on the clearance of vancomycin. ${ }^{5} 6$ The average clearance was $0.309 \mathrm{~L} / \mathrm{h}$ for a neonate with $\mathrm{Scr}$ of $23.3 \mathrm{mmol} / \mathrm{L}$ and body weight of $2.9 \mathrm{~kg}$. No obvious ethnic differences in the clearance of vancomycin were found relative to the earlier studies of Caucasian neonates. Moreover, the established model indicated that in patients with a greater renal clearance status, especially $\mathrm{Scr}<15 \mathrm{mmol} / \mathrm{L}$, current guideline recommendationswould likely not achieve therapeuticarea under the concentration-time curve over24 $\mathrm{h} /$ minimum inhibitoryconcentration $\left(\mathrm{AUC}_{24 \mathrm{~h}} / \mathrm{MIC}\right) \geq 400 .^{3}$ The exceptions to this areBritish National Formulary (2016-2017), Blue Book (2016) and Neofax (2017). Recommended dose regimensfor neonates with differentScrlevelsandpostmenstrual ageswere estimatedbased on Monte Carlo simulations andthe established model.These findings will be valuable for developing individualized dosage regimens in the neonatal ICU setting.

\section{REFERENCES}

1. Abdel HO, Al OS, Nazer LH., Mubarak S, Le, J. Vancomycin pharmacokinetics and predicted dosage requirements in pediatric cancer patients. Journal of Oncology Pharmacy Practice 2015;22(3):448-453 doi: 10.1177/1078155215591386

2. Anderson, B. J., Allegaert, K., In, V. D. A., Cossey, V., \&amp;Holford, N. H. (2007). Vancomycin pharmacokinetics in preterm neonates and the prediction of adult clearance. British Journal of Clinical Pharmacolog;63(1):75-84. doi: 10.1111/j.1365-2125.2006.02725.x

3. Allegaert K, Anderson BJ, Jn, VDA, Vanhaesebrouck, S., \& De, Z. F. (2007). Renal drug clearance in preterm neonates: relation to prenatal growth. Therapeutic Drug Monitoring, 29(3), 284-291. doi: 10.1097/FTD.0b013e31806db3f5

4. Byon, W., Smith, M. K., Chan, P., Tortorici, M. A., Riley, S., \& Dai, H., et al. (2013). Establishing best practices and guidance in population modeling: an experience with an internal population pharmacokinetic analysis guidance. CptPharmacometrics \& Systems Pharmacology,2(7), e51. doi: 10.1016/j.cmpb.2010.04.018

5. Capparelli, E. V., Lane, F. R., Romanowski, G. L., Pharm, M. F., Murray, W., \& Sousa, P., et al. (2001). The influences of renal function and maturation on vancomycin elimination in newborns and infants. Journal of Clinical Pharmacology 41(9), 927-934.

6. Centers for Disease Control and Prevention. (2009). WHO Child Growth Standards. http://www.who.int/childgrowth/en. [EB/OL] 2017-09-12

Disclosure(s) Nothing to disclose 